\title{
Accuracy limits of in-room localisation using RSSI
}

\author{
Francesco Potorti ${ }^{1}$, Alessandro Corucci ${ }^{2}$, Paolo Nepa*2, Francesco \\ Furfari $^{1}$, Paolo Barsocchi ${ }^{1}$, and Alice Buffi ${ }^{2}$ \\ ${ }^{1}$ ISTI, CNR, via Moruzzi 1, I-56124 Pisa \\ ${ }^{2}$ Dept. of Information Engineering, University of Pisa, Pisa, Italy \\ E-mail: Potorti@isti.cnr.it
}

\section{Introduction}

Indoor localisation using inexpensive, non-dedicated wireless devices has spun great attention in the last years. We witness the ubiquitous presence of Wi-Fi access points in offices and homes, soon followed by the increasing spread of low-power, low-cost wireless nodes using the Bluetooth and, in perspective, ZigBee technologies. The presence of a potentially high number of wireless transmitters in indoor spaces has pushed researchers to investigate whether their built-in received signal strength indicator (RSSI) could be exploited to gain information on the relative position of a receiver with respect to a number of transmitters and, by knowing in advance the position of those transmitters, to pinpoint the receiver's position.

Even though RSSI meters are not built to this end, but rather to give information to the higher communication protocol layers about the status of the communication link, their usage is highly attractive, because the information they give is obtained almost "for free". As a consequence, many studies exist which, analytically, through simulations or through real measurements, analyse how a receiver (mobile) can best use RSSI relative to multiple wireless transmitters (anchors) to compute its position $[1,2]$.

In this paper we use detailed ray-tracing simulation to investigate the ultimate performance of indoor, single-room localisation using RSSI measurements in a specific case, and we comment on the results. This study does not lend itself to practical implementation of a localisation method, but rather provides insight into the limits of methods based on RSSI.

\section{Looking at RSSI}

Our environment is an office room at ISTI, CNR, in Pisa. Size is 7.00 by $4.95 \mathrm{~m}$, height is $3.12 \mathrm{~m}$. The room has a single door on the $\mathrm{N}$ wall, a magnetic white-board on the E door, a low metallic cabinet in the SE corner, and a high metal cupboard on the $\mathrm{W}$ wall. The walls are made of gasbeton, the floor is wooden and a lightweight dropped ceiling is in place. The anchors are placed in any of 11 different places at $2 \mathrm{~m}$ height from the floor, some of them with different antenna orientations for a total of 18 anchor configurations (anchors, for short). Both the mobile and the anchors use a $\lambda / 2$ dipole $-\lambda$ being the wavelength at the $2^{\text {nd }}$ channel of the IEEE 802.15.4 standard - which is about $62 \mathrm{~mm}$ long. The power level received from each of the 18 anchors is computed at all the points of a horizontal 156 by 222 grid set at $90 \mathrm{~cm}$ from the floor. The grid meshes are squares with $\lambda / 4$ sides. Figure 1 shows the room and half the grid used. We use a three-dimensional deterministic propagation model based on an inverse ray-tracing algorithm which accounts for

Work partly funded by EU FP6 projects PERSONA (N.045459) and INTERMEDIA (N.38419). 


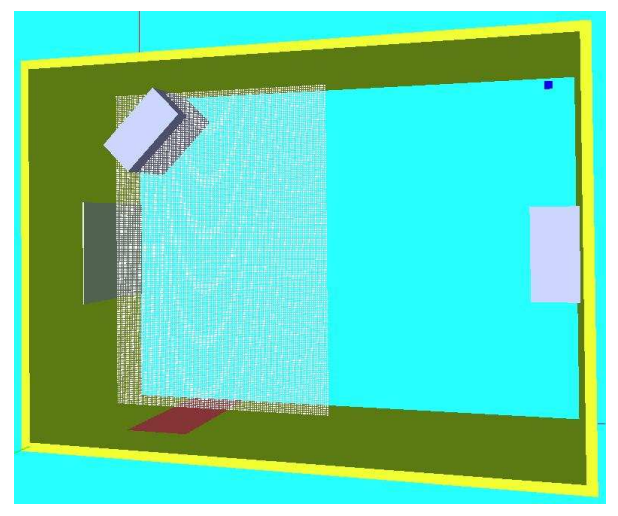

Figure 1: The room environment. Only half of the grid is shown.

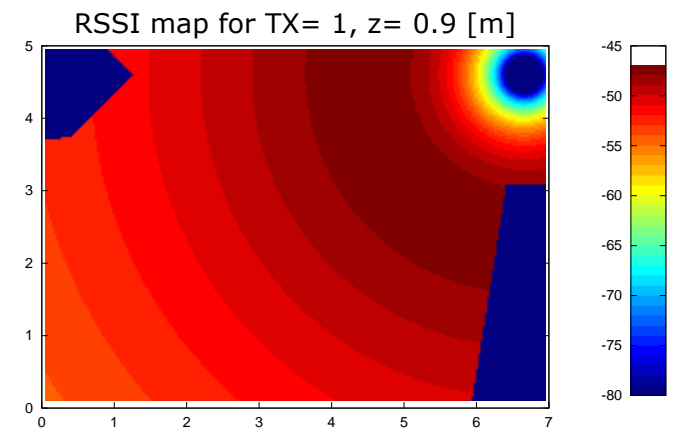

Figure 2: Anchor in a corner, vertical dipole, simulation with no reflections.

contributions up to third order reflections. The model evaluates first-order edge diffractions through heuristic UTD (Uniform Geometrical Theory of Diffraction) dyadic diffraction coefficients, valid for discontinuities on impedance surfaces, and accounts for conductivity and permittivity of the wall materials [3, 4]. The grid is narrow enough that we can assume we have all the information about RSSI on the considered plane. While this scenario is more detailed than most of those assumed in the literature, it still includes many approximations: the presence of wooden tables and chairs is not modelled, nor is the presence of people. Moreover, while ray-tracing is three-dimensional, only a single plane is analysed. In spite of these limitations, we manage to obtain some interesting results.

Let's first pretend that there are no reflections, only the direct ray is considered. Figure 2 shows one instance of such case, with a vertical antenna put at a corner of the room. Observe that the antenna radiation pattern has a strong influence on RSSI pattern. With a vertical antenna, RSSI vs. distance from the antenna is a non-monotone function. If we consider a $1 \mathrm{~dB}$ RSSI resolution for the mobile device, bands of different RSSI values are about $80 \mathrm{~cm}$ wide.

Let's now look at reflections inside the room, and how much they affect the RSSI pattern. Figures 3 and 4 show that the RSSI patterns are indeed very complex, and just moving by few centimetres can change the received value significantly. At the same time, for each given RSSI value, there are many, even far-apart locations in the room where that same value is received. The consequences of these observations are manifold. The following list is an attempt at summarising the most significant ones. In the following, we indicate as localisation error the distance of the computed position from the real one.

- It appears that any attempts at modelling the environment analytically, through the use of simplified path loss formulas, is doomed to fail in a tight environment like this, because reflections dominate the RSSI distribution.

- Even if reflections were not there, the size of the room and the width of the $1 \mathrm{~dB}$ bands would require the mobile a very high RSSI resolution, if one is to obtain a low localisation error using path loss.

- When one abandons path loss formulas and resorts to fingerprinting methods, accuracy of positioning when taking off-line measurements should be a primary 


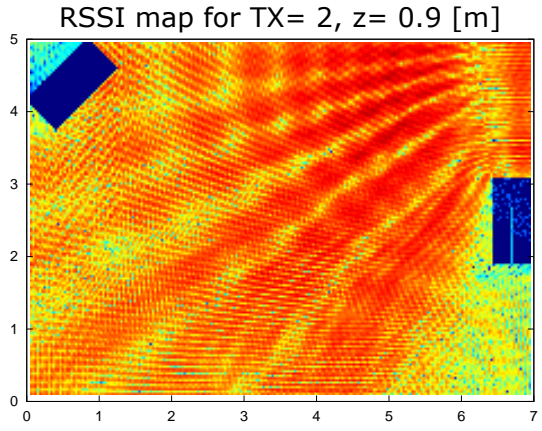

Figure 3: Anchor in a corner, dipole slanted by $45^{\circ}$.

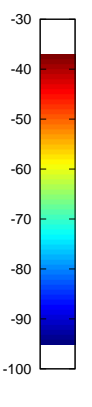

Figure 4: Anchor in the centre of the room, horizontal dipole.

concern: as noted above, errors of few centimetres can significantly change the fingerprint at a given location. Errors can be caused by incorrect measurements in real environments, incorrect modelling in simulated environments, small changes in the environment itself after fingerprinting, and so on.

- Fingerprinting methods using interpolation of grid values assume that the RSSI distribution in the area of interest is somewhat continuous, but by looking at figures 3 and 4, one would say that this is clearly not the case. Even when only nearest-neighbour interpolation is used, the strongly non-linear distribution of RSSI will make this process prone to significant localisation error.

- For any method used, errors both in RSSI measurement at the mobile and power strength at the anchor should be kept into account. Both statistic and systematic errors can occur.

The above list of possible sources of significant localisation error is daunting, to the point of making one wonder whether it is possible at all to obtain any usable result in a single-room environment using RSSI only. The purpose of this paper is to shed some light on this question.

\section{A likelihood approach}

The RSSI values in the room locations are computed using the above mentioned raytracing propagation model and two-dimensional grid. For each point on the grid we set a normal standard positioning error of $10 \mathrm{~cm}$, to account for errors on the room measurement, and we set a normal standard error of $2 \mathrm{~dB}$ on measurements of RSSI from anchors, to account for both receiving and transmitting errors. A mobile receives RSSI information from a number of anchors (an RSSI vector), and for each grid node we compute the likelihood that the mobile is there. For illustration purposes, Figure 5 depicts two typical log-likelihood maps, using the best subset of 4 anchors and all 18 anchors, respectively. In both pictures, the cross represents the computed location, estimated as the centre of mass of the top $10 \%$ log-likelihoods, while the actual location is centre of the circle of small discs, whose radius is twice the standard positioning error.

Actually, Monte Carlo simulation has shown that the ML (maximum likelihood) criterion is more effective than the centre of mass approach; with ML, the computed 

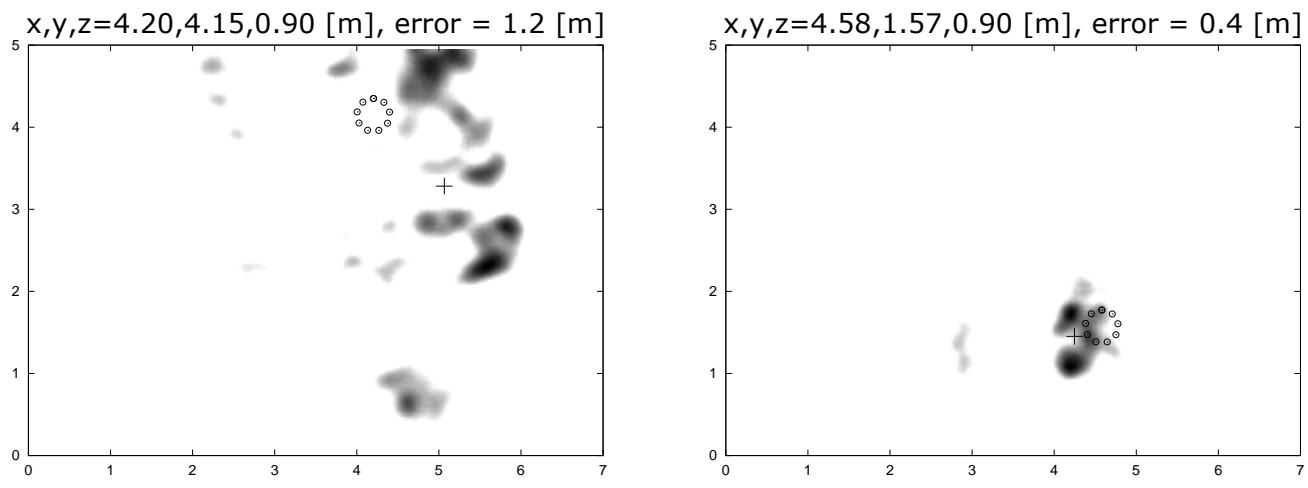

Figure 5: Top 10\% of log-likelihood in shades of gray (darker is higher). Two typical cases for the best 4 anchors (left) and all 18 anchors (right).

location is set at the grid point where the likelihood of receiving the given RSSI vector is maximum. This method is able to exploit all the information gathered from any anchor, and in fact heuristics have shown that, for any given set of anchors, adding one anchor reduces the localisation error, while removing one increases it. Using all 18 anchors, ML produces a median localisation error of only $21 \mathrm{~cm}$ and a third quartile of $73 \mathrm{~cm}$. The third quartiles for the best subsets of 3,5,7 and 12 anchors are 356, 300, 267 and $145 \mathrm{~cm}$, respectively.

\section{Conclusions}

An ML approach is able to exploit all available information, and has proven to be a powerful method to evaluate RSSI localisation methods. We plan to use it to attack problems like optimal anchor placement, exploiting multiple mobile receivers, and localisation coupled to tracking.

One preliminary result is that, when accounting for positioning and RSSI errors in a specific single-room scenario, we needed at least 12 anchors to obtain a third quartile localisation error of less than $150 \mathrm{~cm}$. This suggests that much investigation is needed for single-room RSSI localisation to attain any useful performance.

\section{References}

[1] E. E. X. Li and R. Martin, "The limits of localization using signal strength: a comparative study," in Sensor and Ad Hoc Comm. and Net., 2004, pp. 406-414.

[2] K. Lorincz and M. Welsh, "MoteTrack: a robust, decentralized approach to RFbased location tracking," Pers.Ubiq. Comp., vol. 11, no. 6, pp. 489-503, 2007.

[3] M. Porretta, P. Nepa, G. Manara, and F. Giannetti, "Location, location, location," IEEE Vehicular Technology Mag., vol. 3, no. 2, pp. 20-29, June 2008.

[4] M. Porretta, P. Nepa, G. Manara, F. Giannetti, M. Dohler, B. Allen, and A. H. Aghvami, "A novel single base station location technique for microcellular wireless networks: description and validation by a deterministic propagation model," IEEE Trans. on Vehicular Technology, vol. 53, no. 5, pp. 1502-1514, Sept. 2004. 